\title{
PREVALENCE OF VARIOUS FOCAL LIVER LESIONS AND COMPARISON OF IMAGE QUALITY BETWEEN CONVENTIONAL SONOGRAPHY AND TISSUE HARMONIC SONOGRAPHY IN FOCAL LIVER LESIONS
}

\author{
Senthil Kumar Aiyappan¹, Vadanika V2, Bulabai Karpagam³, S. Vinayagam4, K. C. Saravanan 5
}

${ }^{1}$ Associate Professor, Department of Radiodiagnosis, SRM Medical College and Research Centre, Kattankulathur, Kancheepuram, Tamilnadu.

2Junior Resident, Department of Radiodiagnosis, SRM Medical College and Research Centre, Kattankulathur, Kancheepuram, Tamilnadu.

${ }^{3}$ Associate Professor, Department of Radiodiagnosis, SRM Medical College and Research Centre, Kattankulathur, Kancheepuram, Tamilnadu.

${ }^{4}$ Professor, Department of Radiodiagnosis, SRM Medical College, and Research Centre, Kattankulathur, Kancheepuram, Tamilnadu. 5 Professor, Department of Radiodiagnosis, SRM Medical College, and Research Centre, Kattankulathur, Kancheepuram, Tamilnadu.

ABSTRACT: AIM: To compare the image quality of conventional sonography and tissue harmonic sonography in focal liver lesions and to estimate the prevalence of various focal liver lesions.

MATERIALS AND METHODS: 100 consecutive patients with focal liver lesions, referred to the radiology department over a period of 2 years were enrolled in this study. The final diagnosis of these lesions were confirmed either by CT, MRI or cytology/histopathology. Patients with focal liver lesions were subjected to conventional sonography and tissue harmonic imaging in the same ultrasound machine. Two radiologists who were blinded to the imaging technique used, compared the random images twice. The second evaluation of the same set of images by each observer was done after a minimum interval of 4 months. Results were analysed.

RESULTS: Out of 100 patients, solitary lesions were seen in 36 cases and multiple in 64 cases. Solid lesions were seen in 68 cases and cystic lesions were seen seen in 32 cases. Metastasis was the most common solid lesion and simple hepatic cyst was the most common cystic lesion. Tissue harmonic imaging was better than conventional sonography in assessment of liver lesions.

CONCLUSION: In this study we conclude tissue harmonic imaging is better than conventional sonography in assessment of focal liver lesions and metastasis is the most common solid liver lesion.

KEYWORDS: Tissue Hormonic Imaging; Conventional Sonography; Focal Liver Lesions.

HOW TO CITE THIS ARTICLE: Senthil Kumar Aiyappan, Vadanika V, Bulabai Karpagam, S. Vinayagam, K. C. Saravanan. "Prevalence of Various Focal Liver Lesions and Comparison of Image Quality Between Conventional Sonography and Tissue Harmonic Sonography in Focal Liver Lesions". Journal of Evolution of Medical and Dental Sciences 2015; Vol. 4, Issue 91, November 12; Page: 15633-15636, DOI: 10.14260/jemds/2015/2248.

INTRODUCTION: Focal liver lesions are discrete abnormalities arising within the liver and are frequently detected during imaging of abdomen. They can be classified as developmental, infective, inflammatory, neoplastic or miscellaneous lesions. Recent advances in last few years have provided clinicians with a wide range of choices in imaging modalities, for investigation of patients with suspected focal liver lesions. Various radiological investigations, which can be performed for evaluation of focal liver lesions, include ultrasonography (USG), computed tomography (CT) and magnetic resonance imaging (MRI). USG is the initial non-invasive, simple radiological investigation, done for assessment of liver lesions and plays an important role in reaching final definitive diagnosis of the disease.(1) It is also cost effective and is a widely available modality with no associated radiation hazards.

Financial or Other, Competing Interest: None.

Submission 26-10-2015, Peer Review 27-10-2015,

Acceptance 03-11-2015, Published 10-11-2015.

Corresponding Author:

Senthil Kumar Aiyappan,

\#1880, 4th Street, Vasantham Colony,

Annanagar West, Chennai-600040, Tamilnadu.

E-mail: senthilkumarpgi@yahoo.co.in

DOI:10.14260/jemds/2015/2248.
Apart from conventional sonography (CS), recently new gray-scale sonographic techniques have been developed, which have increased the application of ultrasound in liver imaging. Tissue harmonic imaging is a relatively new sonographic technique, which provides a better image quality than conventional sonography.It is based on phenomenon of non-linear distortion of acoustic signals as they travel through tissues.(2) Until now, many studies have been carried out comparing image quality of one sonographic technique with that of other, in focal liver lesions. In this study we compared the image quality of conventional sonography and tissue harmonic sonography in focal liver lesions and estimated the prevalence of various focal liver lesions in our local population.

MATERIALS AND METHODS: One hundred consecutive patients with focal liver lesions, referred to the radiology department, SRM medical college and research centre over a period of 2 years were enrolled in this study after obtaining permission from institutional ethics committee.

The inclusion criteria were patients with solitary or multiple focal liver lesions, detected during routine grayscale sonography for abdominal complaints, incidentally detected focal liver lesions during CT or MRI of abdomen, screening for hepatocellular carcinoma in patients with underlying cirrhosis. In case of multiple lesions of similar 
was included in the study and in cases of both solid and cystic character, largest lesion lesions, the predominant component of the lesion was considered for evaluation.

Detailed record of history, clinical examination and other relevant investigations of all patients were kept. The procedure was fully explained to the each patient in their language and written informed consent was taken from the patient prior to sonographic examination.

Sonographic examination of focal liver lesions was done on PHILIPS HD (High definition) 11 XE Ultrasonic Machine, using 2-5 MHz curvilinear transducer. Sonography was done by a radiologist with atleast 2 years training in ultrasound. Initially, an overall evaluation of whole of the liver was done with respect to liver size, echotexture, outline, detection of focal liver lesions, their number, topographic localization and relation to adjacent vessels and biliary radicals. For each liver lesion, first conventional sonography was performed, followed by tissue harmonic imaging on the same machine, by using the same transducer. A set of two images were obtained for each focal lesion in liver. These images were then arranged randomly so that the two images of single lesion were not viewed side by side.

Two radiologists who were blinded to the imaging technique used, compared the random images of all focal liver lesions. The second evaluation of the same set of images by each observer was done after a minimum interval of 4 months.

The lesions were evaluated for lesion conspicuity, presence of unwanted artefacts, overall image quality and in cases of cystic lesions presence of internal echoes and posterior acoustic enhancement. Finally considering everything the overall image quality is graded as poor, fair, good and excellent. The final diagnosis of these lesions were confirmed either by CT, MRI or cytology/histopathology.

RESULTS: Evaluation of focal liver lesions was done using conventional sonography and tissue harmonic imaging in 100 patients. Patient recruitment and image acquisition were done prospectively. Later, image analysis was done by two radiologists, independently. The second evaluation of the same set of images by each observer was done after a minimum interval of 4 months. Results are summarized in the form of tables.

DISCUSSION: The radiological investigations performed for evaluation of focal liver lesions, include ultrasonography (USG), computed tomography (CT) and magnetic resonance imaging (MRI). Advances in these modalities have led to improved detection and characterization of focal liver lesions so that a definitive diagnosis can be made non-invasively.(1) CT and MRI play an important role in evaluation of liver lesions, as they provide excellent cross-sectional details of the lesion. However, both of them are not free of limitations. CT is associated with inherent risk of radiation exposure and is a costly investigation.

MRI, though radiation free, is also not as frequently used as ultrasound, because of its high cost. In addition, it is not widely available. Ultrasound is the initial non-invasive radiological investigation, done for assessment of liver lesions and plays an important role.(1) It is also cost effective and is a widely available modality without associated radiation hazards. Recently new gray-scale sonographic techniques have been developed.(3) which have increased the application of ultrasound in liver imaging.

Conventional sonography (CS) is the basic gray scale sonographic technique, which uses the same frequency bandwidth for transmitted as well as received signals. In this technique, the target tissue is examined from single viewing angle only. Therefore, images are degraded by presence of many artifacts, like speckle, clutter and refraction.(2)

Tissue harmonic imaging (THS) is a new sonographic technique, which provides a better image quality than CS.(4)

It is based on phenomenon of non-linear distortion of acoustic signals as they travel through tissues. $(2,4)$ Harmonics are multiples of fundamental or transmitted frequency. Higher harmonic frequencies are generated by propagation of ultrasonic waves through tissues. A bandpass filter is used to process the received signal so that only returning highfrequency harmonics signals are used to form the final image.

Nowadays, only second harmonic or twice the fundamental frequency is used for imaging. Image obtained by THS is better than that produced by CS, due to reduced artifacts, improved signal to noise ratio, enhanced contrast and better spatial resolution.(2-8) THS is particularly useful in obese patients. Limitations of THS are overall coarse echotexture and decreased penetration. $(6,7)$

Previous studies by Yucel C et al.(9) Sodhi K S et al.(10) Choudhry S et al.(11) Kubota K et al.(12) evaluated efficacy of THS as compared to CS and concluded that THS was significantly superior to conventional sonography, especially in obese patients and patients with poor acoustic window. Our study correlated with these previous studies and confirmed that tissue hormonic imaging was better than conventional sonography in assessment of focal liver lesions.

Focal liver lesions are one of the most common abnormalities detected while performing US in patients with suspected abdominal pathology. Focal liver lesions are seen as discrete abnormalities arising within liver, against the background of relatively normal liver parenchyma. Depending on their etiologies, they can be classified as developmental, infective, inflammatory, neoplastic or miscellaneous. Metastasis is the most common liver malignancy as in our study and correct detection of liver metastasis is critical in patient management.(13,14) Hemangiomas are the most common benign liver solid lesion as in our study.

The prevalence of hemangiomas ranges from $1 \%-20 \%$, showing a female predilection. Benign Cystic lesions of liver are common as in our study and may represent a broad spectrum of entities. The differential diagnosis for simple cysts includes benign developmental hepatic cysts, biliary hamartomas, foregut cysts, Caroli disease, and adult polycystic liver disease.(13,14) Our study correlated with the previous studies.(14) in calculating the prevalence of focal liver lesions.

CONCLUSION: Ultrasound is a simple radiological investigation for noninvasive assessment of focal liver lesions. Recent advances in ultrasound techniques have 
made it more accurate and reliable. In this study we have proved that tissue hormonic imaging is better than conventional sonography for assessment of liver lesions.

\section{REFERENCES:}

1. Harvey C J, Albrecht. Ultrasound of focal liver lesions. Eur Radiol 2001; 11:1578-1593.

2. Merritt C R B. Updates in ultrasonography. Radiol Clin North Am 2001; 39:1-10.

3. Claudon M, Tranquart F, Evans D H, Lefevre F, Correas J M. Advances in ultrasound. Eur Radiol 2002; 12:7-18.

4. Kubota K, Hisa N, Nishikawa T, Ohnishi T, Ogawa Y, Yoshida S. The utility of tissue harmonic imaging in liver: a comparison with the conventional gray-scale sonography. Oncol Rep 2000; 7:767-771.

5. Shapiro RS, Wagriech J, Parsons RB, Stancato-Pasik A, Yeh HC, Lao R. Tissue harmonic imaging sonography: evaluation of image quality compared with conventional sonography. Am J Roentgenol 1998; 171:1203-1206.

6. Dresser $\mathrm{T}$ S, Jeffery R B. Tissue harmonic imaging techniques: physical principles and clinical applications. Semin Ultrasound CT MRI 2001; 22: 1-10.

7. Lencioni B, Coini D, Bartolozzi C. tissue harmonic and contrast specific imaging: back to gray scale in ultrasound. Eur Radiol 2002; 12: 151- 165.

8. Tanaka S, Oshikawa O, Sasaki T, Ioka T, Tsukuma H. Evaluation of tissue harmonic imaging for diagnosis of focal liver lesions. Ultrasound Med Biol 2000; 26: 183187.

\begin{tabular}{|c|c|c|}
\hline Sl. No. & Age Distribution (Yr.) & Number \\
\hline 1 & $<10$ & 1 \\
\hline 2 & $10-20$ & 2 \\
\hline 3 & $21-30$ & 10 \\
\hline 4 & $31-40$ & 12 \\
\hline 5 & $41-50$ & 29 \\
\hline 6 & $51-60$ & 21 \\
\hline 7 & $61-70$ & 17 \\
\hline 8 & $71-80$ & 6 \\
\hline 9 & $81-90$ & 2 \\
\hline \multicolumn{2}{|c|}{ Table 1: Age distribution of patients (n=100) } \\
\hline
\end{tabular}

Table 1 shows the age distribution of 100 patients with focal liver lesions, enrolled in our study. Mean age was 49 years (range 8 - 84 years).

\begin{tabular}{|c|c|c|}
\hline Sex & Number & Percentage \\
\hline Male & 42 & $42 \%$ \\
\hline Female & 58 & $58 \%$ \\
\hline \multicolumn{2}{|c|}{ Table 2: Sex distribution of patients (n=100) } \\
\hline
\end{tabular}

Table 2 shows the sex distribution of patients enrolled in the study. There were $42 \%$ males and $58 \%$ female patients in our study.

\begin{tabular}{|c|c|c|}
\hline Nature & Number & Percentage \\
\hline Solid & 68 & $68 \%$ \\
\hline Cystic & 32 & $32 \%$ \\
\hline \multicolumn{2}{|c|}{ Table 3: Nature of hepatic lesions (n=100) } \\
\hline
\end{tabular}

In our study, the most common malignant liver lesion was metastasis, most common benign solid lesion was hemangioma and most common benign cystic lesion was simple hepatic cyst.

9. Yucel C, Ozdemir H, Asik E, Isik S. Benefits of tissue harmonic imaging in evaluation of abdominal pelvic lesions. Abdom Imaging; 28:103-109.

10. Sodhi KS, Sidhu R, Gulati M, Saxena A, Suri S, Chawla Y. Role of tssue harmonic imaging in focal hepatic lesions: comparison with conventional sonography. J Gastroentrol Hepatol 2005; 20:1488-1493.

11. Chaudhry S, Gorman B, Charboneau JW, Tradup DJ, Beck RJ, Kofler JM et al. Comparison of tissue harmonic imaging with conventional US in abdominal disease. Radiographics 2000; 20:1127-1135.

12. Kubota $\mathrm{K}$, Hisa N, Nishikawa T, Ohnishi T, Ogawa $\mathrm{Y}$, Yoshida S. The utility of tissue harmonic imaging in liver: a comparison with the conventional gray-scale sonography. Oncol Rep 2000; 7:767-771.

13. Matos AP, Velloni F, Ramalho $M$, AlObaidy $M$, Rajapaksha A, Semelka RC. Focal liver lesions: Practical magnetic resonance imaging approach. World J Hepatol. 2015 Aug 8; 7(16):1987-2008.

14. Horta G, López M, Dotte A, Cordero J, Chesta C, Castro A, Palavecino P, Poniachik J. Benign focal liver lesions detected by computed tomography: Review of 1,184 examinations. Rev Med Chil. 2015 Feb; 143(2):197202.

Table 3 shows nature of focal liver lesions included in the study. Out of 100 cases included in the study, solid lesions were the commoner form of hepatic lesions, constituting $68 \%$ of all cases. Cystic liver lesions were found in $32 \%$ patients.

\begin{tabular}{|c|c|c|}
\hline Focality of lesions & Number & Percentage \\
\hline Solitary & 36 & $36 \%$ \\
\hline Multiple & 64 & $64 \%$ \\
\hline \multicolumn{2}{|r|}{ Table 4: Focality of liver lesions (n=100) } \\
\hline
\end{tabular}

Table 4 shows distribution of hepatic lesions with respect to their number. Of all 100 lesions, 36\% lesions were solitary, while in $64 \%$ cases, multiple hepatic lesions were noted.

\begin{tabular}{|c|c|c|c|}
\hline $\begin{array}{c}\text { Sl. } \\
\text { No }\end{array}$ & $\begin{array}{c}\text { Type of } \\
\text { hepatic lesion }\end{array}$ & Number of cases & Percentage \\
\hline $\mathbf{1 .}$ & Metastasis & 48 & $48 \%$ \\
\hline $\mathbf{2}$ & Simple cyst & 20 & $20 \%$ \\
\hline $\mathbf{3 .}$ & Hemangioma & 14 & $14 \%$ \\
\hline $\mathbf{4 .}$ & $\begin{array}{c}\text { Hepatocellular } \\
\text { carcinoma }\end{array}$ & 10 & $10 \%$ \\
\hline $\mathbf{5 .}$ & Abscess & 5 & $5 \%$ \\
\hline $\mathbf{6 .}$ & Hydatid cyst & 3 & $3 \%$ \\
\hline \multicolumn{3}{|c|}{ Table 5: Spectrum of hepatic lesions (n=100) } \\
\hline
\end{tabular}

Table 5 shows spectrum of focal liver lesions seen in the study. Hepatic metastasis was the commonest focal 
abnormality detected in liver, comprising $48 \%$ of all lesions. Most common benign focal solid lesion was hemangiomas and cystic lesion was simple hepatic cyst comprising $34 \%$ of all lesions.

IMAGE ANALYSIS: All 100 focal liver lesions were evaluated for lesion conspicuity, artifact elimination, posterior acoustic enhancement, presence of internal echoes and finally for overall image quality. Overall image quality is graded as poor, fair, good and excellent.

\begin{tabular}{|c|c|c|c|c|}
\hline $\begin{array}{c}\text { Imaging } \\
\text { modality }\end{array}$ & Poor & Fair & Good & Excellent \\
\hline $\mathrm{CS}$ & $24.2 \%$ & $42.1 \%$ & $30.7 \%$ & $3 \%$ \\
\hline $\mathrm{THS}$ & $15.5 \%$ & $43.2 \%$ & $32.5 \%$ & $8.6 \%$ \\
\hline \multicolumn{6}{|c|}{$\begin{array}{c}\text { Table 6: Grading of focal liver lesions using } \\
\text { conventional sonography and tissue hormonic } \\
\text { imaging }\end{array}$} \\
\hline
\end{tabular}

Table 6 shows tissue hormonic imaging performed better than conventional sonography with most of them falling in fair, good or excellent category and only $15.5 \%$ showed poor grading.

Fig. 1: 50-year-old male patient with simple hepatic cyst in liver. A) Conventional sonography shows moderately obscured margins of the cyst (White arrows) B) Corresponding tissue hormonic image showing well-defined margin and posterior acoustic enhancement of the cystic lesion (White arrows).

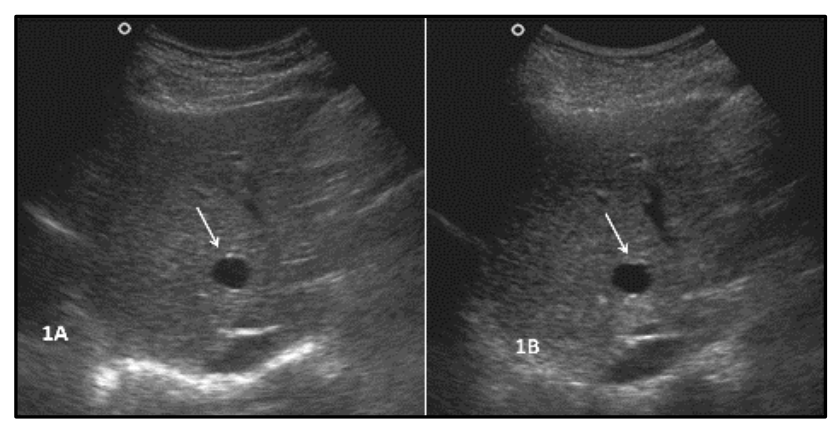

Fig. 1
Fig. 2: 65-year-old female with liver metastasis A) Conventional sonography image shows presence of a hypoechoeic lesion in segment IV A of liver. Margins of the lesion are ill defined in this image (White arrows). B) Corresponding tissue hormonic image shows well defined margin of this lesion with good overall image quality (White arrows).

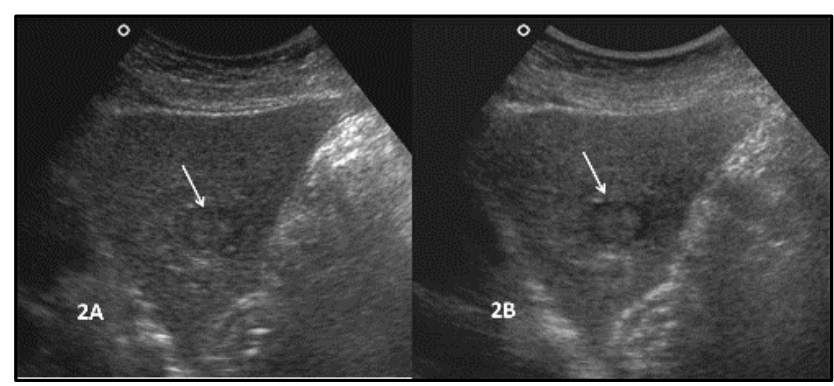

Fig. 2

Fig. 3: 22-year-old with liver hemangioma A) Conventional sonography image shows presence of a hyperechoeic lesion (White arrows). B) Corresponding tissue hormonic image shows well defined clear margin of this lesion with excellent image quality (White arrows).

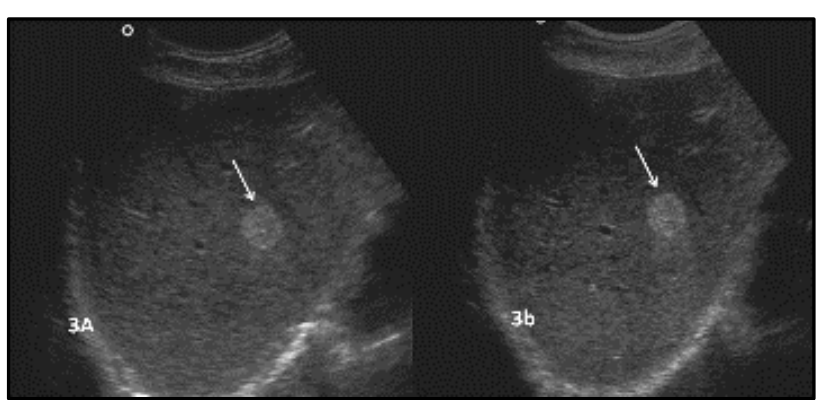

Fig. 3 